\title{
Increasing work readiness of fresh IT graduates at interviews: A scoping review
}

\author{
Dulmini Aeheliyagoda ${ }^{\# 1}$, Suranji Nadeeshani ${ }^{\# 2}$ \\ ${ }^{\# 1}$ Department of IT, CINEC Campus \\ Millennium Drive, IT Park, Malabe, Sri Lanka. \\ ${ }^{2}$ suranjidcinec. edu
}

\begin{abstract}
Interviews are a widely used method in which the performance and knowledge of potential employees are evaluated. Fresh graduates who come out to the job industry are rarely armed with prior experience in facing a job and securing employment, which leads to them underperforming at actual interviews. The lack of exposure to an interviewing environment together with inadequate preparation is the main reason for such underperformance. This paper examines the short-comings associated with graduate interviews and attempts to identify the predominant factors that would contribute to an improvement in performance. It then aims to propose a solution in terms of a computer based interviewing platform to solve this problem.
\end{abstract}

\section{CCS CONCEPTS}

- Human Computer Interaction (HCI); Artificial Intelligence; Speech Synthesis

Keywords - Qualitative Evaluation, Scoping Review Interviews, Employment, Fresh Graduates

\section{INTRODUCTION}

Two million students graduate from universities each year [1] but roughly, only $65 \%-70 \%$ of them manage to obtain jobs after graduation [1]. Research carried out into this area show recession, lack of qualifications and unrealistic expectations [2] as being the main reasons for such overall unemployment in the world. However, there is accumulating evidence in terms of a fresh graduate, indicating the principal reason for unemployment to be under- performance at interviews [3].

An interview acts as a method through which a potential employer gauges the suitability of a student in terms of employment. Factors such as confidence, punctuality, together with knowledge are the deciding factors behind success at an interview. These characteristics are measured by questioning the students on various aspects in relation to the job that they apply for. The inability of a student to successfully answer and portray a demeanour of confidence at an interview results in the student being deemed unsuitable for employment.

Very few studies and system implementations carried out to identify evidence targeted towards improving performance at interviews exist. Moreover, these studies included no in-depth investigation on fresh graduates and focused mainly on job holders. Thus, this short paper seeks to evaluate and propose a method to improve the performance of fresh graduates at interviews thereby reducing their unemployment rates, with emphasis on human- human and human- computer interactions.

\section{METHODOLOGY}

Pope, Ziebland and Mays [4] propose a scope review as an effective means of analyzing qualitative data in terms of highlighting relevant literature in a particular research

area. Such reviews are useful in identifying the main sources and types of information available especially in areas where little might be known. It was decided that the scoping review based on Arksey and O'Malley's framework [5- 6] was appropriate to meet the objectives of this study.

\subsection{Research Questions}

This study answered the following question: What factors from literature contribute to improvement of the performance of fresh graduates, at interviews?

\subsection{Relevant Studies}

The author searched online electronic databases such as Google and Google Scholar for articles published between 1990 and 2016. Initial keywords were used to capture the primary factors that affected performance at interviews. See Table 1 for search terms.

Table 1: Examples of Search Terms

\begin{tabular}{ll}
\hline Search Terms & \\
\hline "performance" & "interviews" \\
"fresh graduates" & "employment rates" \\
"job interview" & "interviewing platforms" \\
\hline
\end{tabular}

Several industry specialists were selected to be interviewed to gather first- hand industry based information related to how interviews are conducted for fresh graduates and to discover how they fared at such interviews. The interviews were to be either one-to-one interviews or telephone interviews.

Questionnaires were used as an additional means of obtaining information directly from previous fresh graduates as to the type of questions they faced at their first interviews; their experience and their ideas on what they thought would have been worth knowing going into their first interview. 
The use of several such methods to collect data covers all aspects and participants' points of view thus increasing visibility of the research and helps focus on outcomes expected from a fresh graduate during their first interview in terms of both the employer and the interviewee.

\subsection{Study Selection}

\subsubsection{Peer-reviewed studies.}

Peer- reviewed studies, regardless of their design, met the inclusion criteria if they contained information on the search terms included within Table 1. Studies were excluded if they were theoretical in nature and if they were not relevant to the Information Technology (IT) industry.

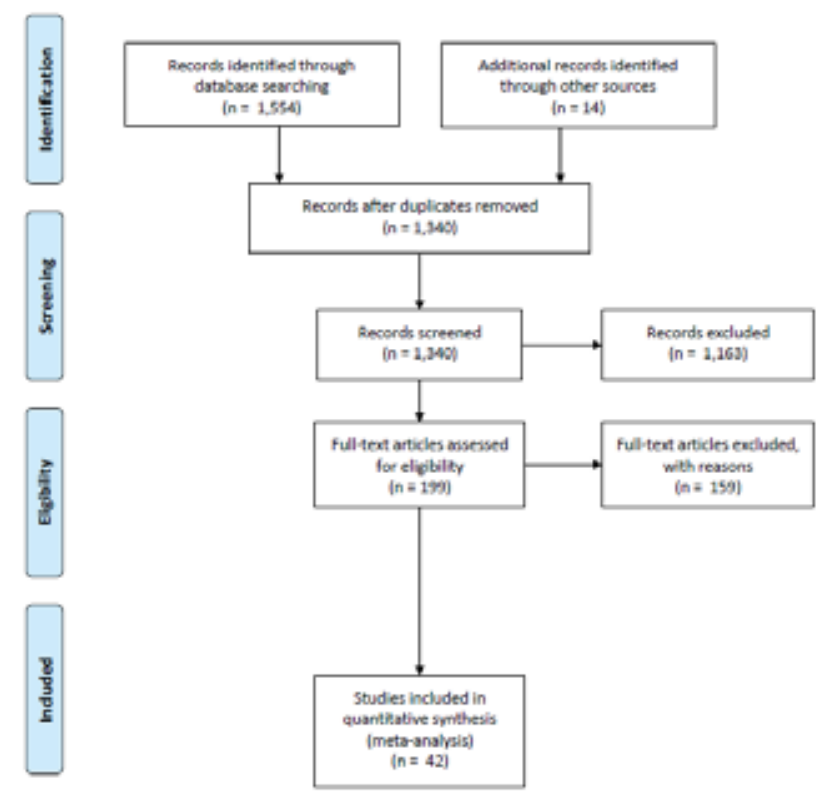

Figure 1: PRISMA Flow diagram for article selection

\subsubsection{Interviews.}

Similarly, a random sample of employers in IT companies within Sri Lanka were initially selected to be interviewed. Companies that were not IT related were excluded. However, the study extended to various departments within the companies such as the Human Resources Department, the Technical Department, etc. within which an interview is conducted.

\subsubsection{Questionnaires.}

Questionnaires were prepared, reviewed by two peers and were sent out to a random sample of thirty. The questions covered aspects related to identifying the personal experience of a person's first interview fresh out of university and their personal opinions. Only the population encompassing the IT sector were to be considered for this study.

\subsection{Chart Data}

Data was extracted from the articles, interviews with industry personnel and questionnaire responses.

\subsection{Collate Summarize Report}

The author collectively compared the data to characterize the research literature while also using questionnaire and industry interview findings to identify the breadth and gaps within the study, using descriptive statistics.

\section{RESULTS}

\subsection{Peer-reviewed studies}

The initial search yielded 1,554 articles with 241 duplicates resulting in 1,340 distinct potential articles. The articles were then screened by two reviewers and 1,298 removed. Of these, $13 \%$ addressed job holders, $20 \%$ professions other than IT and the remaining were purely theoretical. Forty-two of the identified articles met the inclusion criteria. They included studies with discussions on methods/ systems used to improve performance at interviews, (more). There was a notable absence of literature specifically aimed at fresh graduates.

\subsection{Interviews}

Interviews were to be conducted with ten industry specialists however only six of them could be contacted and after the data collected was analyzed, four of the interviews were selected. The two that were excluded did not contain sufficiently detailed information and/ or were missing responses.

\subsection{Questionnaires}

Questionnaires were sent out to thirty randomly selected individuals out of which twenty responses were received. Ten of the responses were excluded due to missing values and inappropriate answers.

\section{DISCUSSION}

\subsection{Assessment in graduate selection}

University graduates are a key source of new recruits for organizations enabling organizations to meet the need for trainable and highly educated individuals that bring a fresh perspective and new ideas into the organization. The measures of organizational effectiveness highlight that identification and selection of the best candidates depend on past achievement and range of ability [7].

Various methods of candidate assessment for recruitment exist, however, several studies have supported the use of interviews as the most appropriate selection mechanism for recruitment of candidates [8-9]. The two most predominant interview mechanisms available are structured and unstructured interviews. Findings suggest that structured interviews are seen to be more suited as a 
means of recruitment due to the ability to define content explicitly and; the ability to replicate and standardize an interview [10]. It also tends to be the popular choice with $40 \%$ of interview participants expressing so.

The aspects that determine success at an interview in terms of obtaining the best output for an organization in additional to technical knowledge; consist of speaking skills, demonstration of confidence, social behavior, and body language.

\subsection{Complications associated with graduate interviews}

A comprehensive review indicates that several factors render graduate interviews to be particularly difficult. The most significant factor elevating the complications with graduate interviews is that most graduates possess almost no direct job-related experience (reference). The lack of such experience is shown to have a direct impact on performance since even entry- level positions require a certain amount of background knowledge.

$80 \%$ of graduates that answered the questionnaire agreed that even though they are exposed to verbal communication and presentations during their time at University, it does not provide sufficient preparation to match the terms of oral assessment related to interviews. Thus, graduates may not fully possess the confidence to articulate their knowledge and skills at an interview. This was further confirmed by the data collected during the industry interviews where all employers confirmed that graduates tended to have difficulty in articulating their knowledge at interviews. Further, questioning revealed nervousness to be the chief cause of shortcomings associated with communication between graduates and potential employers.

The next most significant constituent that poses a threat to graduates at an interview is the tendency of the employer to judge a candidate based on social cues such as body language, tone of voice and intonation [11], which although it may enhance value of the verbal answer provided by a candidate, can prove to tip the scale if candidates are not prepared and are nervous.

Despite the growth of recruitment of graduates through interviews, relatively little is known about the methods used to assess and select graduates to positions within an organization. The fact that graduates lack previous experience and tend to be nervous raises questions in relation to how Universities can use technology to assist in increasing employment rates of fresh graduates. A review of already existing software systems with a particular focus on interviews follows.

\subsection{Factors identified to improve performance at interviews}

\subsubsection{Pre-preparation in relation to industry segmentation}

All findings highlighted pre-preparation as key to high performance at interviews regardless of applying as a fresh graduate or as a candidate with previous work experience. According to the survey responses, this factor was ranked highest with $80 \%$ in agreement when compared to the other factors (age, experience, gender, personality, appearance, attitude and cultural fit).

Preparation differs based on the functional segment of the industry chosen by a candidate. For example, the method of preparation for a role as a Software Engineer would differ from that of a Network Engineer even though both roles reside within the same industry. In addition, the most commonly listed domains of specialization were Software Engineer (58\%), Business Analyst (42\%) and Network Engineer (32\%).

\subsubsection{Confidence Interview}

Findings based on employer feedback identified that confidence through body language during response was ranked second highest.

\subsubsection{Gender}

The paper by Behrend et al. [12] conveys that the appearance of an interviewee's avatar has an influence on the rating of his performance. This is further confirmed with the findings of the questionnaires where $68 \%$ of respondents had agreed that the gender of an interviewer depended on the level of confidence they portrayed at the interview.

\subsubsection{Experience}

Although the most significant factor with relation to interview performance usually tends to be previous work experience, this factor had the lowest rating with only $10 \%$ of all respondents; interviews and questionnaires, in agreement since fresh graduates usually tend to have no previous experience with regard to the jobs they apply for after graduation.

\subsection{Existing systems used in interviews}

Most related work on rule based systems incorporating human computer interaction has been done in the fields of natural language processing [13] and machine learning [14]. However, Clark et al. [15] has experimented with developing a Question- Answering system merging online- text, with a knowledge base, which infers answers based on the questions posed, at- real time. This knowledge base approach uses a set of rules to carryout induction and arrives at a conclusion based on the facts and the rule extracted. We propose to use a similar concept formed around a set of 'if-else' rules to fire questions based on rules inferred.

There has been a gradual exploration in mimicking human behavior within computer based systems during the past few years [16], thereby allowing users to directly communicate and interact through a graphical representation of themselves such as Avatars. Behrend et al. [12] have used this abstraction to show that the appearance of an interviewee's avatar has a positive influence on the rating for his performance. The virtual software platform 'Second Life' [17] is based on a similar 
concept whereby it acts as a 3D interactive world within which a virtual job fair can be conducted. The simulated virtual environment within Second Life allows both an employer and an interviewee to create computergenerated images representing themselves to conduct or participate in an interview. While it shows that such fully virtual platforms are beneficial for interviewees, due to the experience being less daunting, employers tend to be unsatisfied with the outcomes of such interviews due to the inability to read an applicant's body language. This suggests that a platform where only employers are represented as avatars may bring the best of both worlds. Research findings dictate that a system functioning on the basis of usability in terms of human computer interaction to eliminate the complications identified within this study to assist in providing their students with the experience required to attain optimal performance at an interview.

\section{CONCLUSIONS}

This scoping reveals several gaps in literature. This is also evident in terms of the studied population since the focus on most studies was directed towards improving interview performance of job holders. The majority of quantitative studies were cross-sectional in nature portraying a need for more in-depth interventional studies. The study population mainly encompassed of IT graduates and thus more research is needed to expand the study to other industry sectors.

Other gaps were highlighted by the way existing systems handled interview systems where not all factors identified to improve performance were included in a single system. Each system contained one or more aspects that could counter-act the complications identified but not one system could eliminate all factors at once.

Finally, our findings suggest that little literature attempt an interaction between computers and humans to mimic the environment of an interview to ease nervousness. Further examination of criteria is needed; notably in developing and testing a system that could eliminate all complications identified within this study.

\section{REFERENCES}

[1] Max Roser and Esteban Ortiz-Ospina. 2018. Tertiary Education. Retrieved August 12, 2018 from https://ourworldindata.org/tertiary-education

[2] Melissa S Cardon and Christopher E Stevens. Managing human resources in small organizations: What do we know? 2004. Human Resource Management Review 14, 3 (2004), 295-323. DOI: https://doi.org/10.1016/j.hrmr.2004.06.001

[3] Helen Atkinson \& Martin Pennington. 2012. Unemployment of engineering graduates: the key issues. Engineering Education. 7,2, (2012) 7-15. https://doi.org/10.11120/ened.2012.07020007

[4] Pope, C., Ziebland, S. \& Mays, N., 2000. Analysing Qualitative Data. Qualitative Research in Health Care, BMJ, 320 (2000) 6381. http://dx.doi.org/10.1002/9780470750841.ch7
[5] Danielle Levac, Heather Colquhoun, and Kelly K O'Brien. 2010. Scoping studies: advancing the methodology. Implementation Science, 5(1). https://doi.org/10.1186/1748-5908-5-69

[6] Heather L. Colquhoun, Danielle Levac, Kelly K. O'Brien, Sharon Straus, Andrea C. Tricco, Laure Perrier, Monika Kastner, David Moher. Scoping reviews: time for clarity in definition, methods, and reporting. Journal of Clinical Epidemiology 67, 12 (2014), 1291-1294. https://doi.org/10.1016/j.jclinepi.2014.03.013

[7] Brian S. O'Leary, Mary Lou Lindholm, Richard A. Whitford, Stephen E. Freeman. Selecting the best and brightest: Leveraging human capital. Human Resource Management 41, 3 (2002), 325340. https://doi.org/10.1002/hrm.10044

[8] Richard A. Posthuma, Frederick P. Morgeson, Michael A Campion. Beyond Employment Interview Validity: A Comprehensive Narrative Review of Recent Research and Trends Over Time. Personnel Psychology 55, 1 (2002), 1-81. https://doi.org/10.1111/j.1744-6570.2002.tb00103.x

[9] Therese Macan. The employment interview: A review of current studies and directions for future research. Human Resource Management Review 19, 3 (2009), 203-218. https://doi.org/10.1016/j.hrmr.2009.03.006

[10] Rebecca L. Jackson, Michael J. Vitacco. Structured and Unstructured Interviews. Ziskin's Coping with Psychiatric and Psychological Testimony, (2012), 302-310. https://doi.org/10.1093/med:psych/9780195174113.003.0015

[11] Debi Laplante and Nalini Ambady. On How Things Are Said. Journal of Language and Social Psychology 22, 4 (2003), 434-441. https://doi.org/10.1177/0261927x03258084

[12] Tara Behrend, Steven Toaddy, Lori Foster Thompson, and David J. Sharek. 2012. The Effects of Avatar Appearance on Interviewer Ratings in Virtual Employment Interviews. Computers in Human Behavior. 28, $6 \quad$ (2012), 2128-2133. https://doi.org/10.1016/j.chb.2012.06.017

[13] Bassam Hammo, Hani Abu-Salem, and Steven Lytinen. 2002. QARAB: A Question Answering System to Support the Arabic Language. Proceedings of the ACL-02 workshop on Computational approaches to semitic languages - (2002). DOI: https://doi.org/10.3115/1118637.1118644

[14] Junwei Bao, Nan Duan, Ming Zhou, and Tiejun Zhao. 2014. Knowledge-Based Question Answering as Machine Translation. Proceedings of the 52nd Annual Meeting of the Association for Computational Linguistics (Volume 1: Long Papers) (2014). DOI: https://doi.org/10.3115/v1/P14-1091

[15] Peter Clark, John Thompson and Bruce Porter. 1999. A Knowledge-Based Approach to Question-

Answering. In the AAAI Fall Symposium on Question-Answering Systems, CA: AAAI. (March 1999) 43-51

[16] David A. Bray and Benn R. Konsynski. 2007. Virtual worlds. ACM SIGMIS Database 38, 4 (2007), 17. DOI: https://doi.org/10.1145/1314234.1314239

[17] Kaleriya Zelenskaya and Neha Singh. 2011. Exploring Virtua Recruiting From Employers' Perspective Using "Second Life". Journal of Human Resources in Hospitality \& Tourism. 10, 2. (October 2011). 117-128. DOI: https://doi.org/10.1080/15332845.2011.536505 